ARTICLE

Received 7 Jun 2015 | Accepted 9 Oct 2015 | Published 10 Nov 2015

\title{
Hybrid graphene plasmonic waveguide modulators
}

\author{
D. Ansell, I.P. Radko², Z. Han², F.J. Rodriguez' ${ }^{1}$ S.I. Bozhevolnyi ${ }^{2} \&$ A.N. Grigorenko ${ }^{1}$
}

The unique optical and electronic properties of graphene make possible the fabrication of novel optoelectronic devices. One of the most exciting graphene characteristics is the tunability by gating which allows one to realize active optical devices. While several types of graphene-based photonic modulators have already been demonstrated, the potential of combining the versatility of graphene with subwavelength field confinement of plasmonic waveguides remains largely unexplored. Here we report fabrication and study of hybrid graphene-plasmonic waveguide modulators. We consider several types of modulators and identify the most promising one for telecom applications. The modulator working at the telecom range is demonstrated, showing a modulation depth of $>0.03 \mathrm{~dB}_{\mu} \mathrm{m}^{-1}$ at low gating voltages for an active device area of just $10 \mu \mathrm{m}^{2}$, characteristics which are already comparable to those of silicon-based waveguide modulators while retaining the benefit of further device miniaturization. Our proof-of-concept results pave the way towards on-chip realization of efficient graphene-based active plasmonic waveguide devices for optical communications.

\footnotetext{
${ }^{1}$ School of Physics and Astronomy, University of Manchester, Manchester M13 9PL, UK. ${ }^{2}$ Centre for Nano Optics, University of Southern Denmark, Campusvej 55, DK-5230 Odense M, Denmark. Correspondence and requests for materials should be addressed to A.N.G. (email: sasha@manchester.ac.uk).
} 
G raphene holds a great potential to provide efficient graphene-based photodetectors ${ }^{1-4}$, dedicated sensors ${ }^{5-7}$ and various optoelectronic devices ${ }^{8-10}$. Tunability of graphene conductivity by gating ${ }^{11-14}$ should allow one in principle to realize compact optical modulators. While several types of graphene-based photonic modulators have already been demonstrated $^{15-19}$, the combination of plasmonic waveguides with graphene for the task of light modulation remains elusive. Hybrid graphene modulators ${ }^{15-19}$ promise unrivalled speed, low driving voltage, low power consumption and small physical footprints. Such modulators will be welcomed by telecommunications and optoelectronics. It is commonly assumed that the exceptional properties of graphene-based modulators are because of the fact that graphene is a material described by many superlative characteristics ${ }^{20}:$ it is the thinnest, the strongest, with record thermal conductivity, showing the highest current density, completely impenetrable even for helium and with the highest charge mobility. Some of these extraordinary parameters, however, come about from dividing graphene characteristics by its extremely small thickness; a trick one performs to compare two-dimensional graphene with threedimensional materials. In optics, for example, relatively small graphene absorption of $\sim 2.3 \%$ (observed at normal light incidence and defined by the fine structure constant ${ }^{14}$ ) could potentially result in extremely high modulation coefficient of $300 \mathrm{~dB} \mu \mathrm{m}^{-1}$ (when exploiting optical Pauli blocking') after normalization by graphene thickness, $t_{\mathrm{gr}}=0.335 \mathrm{~nm}$, is applied.

The small graphene thickness, while being great for obtaining superlative numbers, represents a significant hindrance for the tasks of active control of optical devices, since it implies that one graphene layer can affect only a small volume of propagating radiation, resulting in relatively small absorption and hence modulation induced by gating. In the transverse modulator configuration (that is, when radiation is incident on and transmitted through a graphene layer), the achievable modulation depth is just $\sim 0.1 \mathrm{~dB}$ per graphene layer. For waveguide modulators, in which a waveguide mode is propagating along and confined near a graphene plane (and which are the most promising for on-chip information processing), the portion of the waveguide mode interacting with the graphene layer is very limited ${ }^{15}$, depending strongly on the mode confinement. The resulting mode absorption coefficient (that represents the maximum modulation coefficient achievable via Pauli blocking) can be roughly estimated as follows: $A \sim \frac{0.2 n \gamma g^{2}}{\lambda} \mathrm{dB} \mu \mathrm{m}^{-1}$, where $n$ is the waveguide effective refractive index, $\gamma$ is the geometrical coverage factor, $\lambda$ is the free space wavelength and $g=E_{\text {in }} / E_{\mathrm{a}}$ represents the in-graphene-plane field component (responsible for the absorption by graphene) weighted with respect to the mode field (Supplementary Discussion 1). For typical parameters, $A$ is at the level of $0.02-0.2 \mathrm{~dB}_{\mu \mathrm{m}}^{-1}$ in the general case of a hybrid graphene-waveguide modulator. Several graphene layers separated by dielectric can be used to improve this number ${ }^{21}$. However, it is difficult to arrange efficient gating for such a system and active optical devices normally involve a maximum of two graphene layers ${ }^{17}$.

To increase graphene interaction with light, one can use conventional metal plasmonics $3,9,22-26$. Plasmonic waveguides could provide smaller volumes of propagating modes (larger effective $n$ ) and local field enhancement (large values of $g$ ), which would result in higher modulation depth. There are two main problems in combining graphene and plasmonic waveguides to achieve optical modulation by gating: (i) the presence of metal layers complicates the task of gating with the spacer dielectrics being often affected by both high electric fields ${ }^{27}$ and light ${ }^{28}$ and (ii) it is difficult to realize plasmonic waveguide modes with large in-plane field components, since fields of surface plasmon polaritons (SPPs) supported by metal-dielectric interfaces are predominantly transverse in dielectrics ${ }^{27}$ (and the perpendicular electric fields do not excite currents in recumbent graphene). While the latter feature provides a useful possibility to safeguard metal plasmonics with graphene without degrading plasmon characteristics $^{24}$, it makes the endeavour of designing hybrid graphene plasmonic waveguide modulators (HGPWMs) rather challenging-yet not hopeless. Simple estimations indicate the principal possibility of achieving strong SPP absorption by the graphene monolayer (even at telecom wavelengths), with the absorption coefficients reaching up to $\sim 2 \mathrm{~dB}_{\mu \mathrm{m}}^{-1}$ for strongly confined SPP modes (Supplementary Discussion 1B,C). At the same time, one should bear in mind that the SPP absorption by the graphene monolayer turns out to be quite similar in magnitude to that by adjacent metal because of Ohmic loss, as both absorption mechanisms are essentially determined by the same ratio between the transverse electrical field component in dielectric and the longitudinal one (in metal or graphene). To circumvent this very problematic issue (Supplementary Discussion 1), we suggest breaking natural coplanarity of graphene and metal layers so as to allow strong transverse SPP field components to have non-zero projections on a graphene layer.

Here we report the first HGPWM realization using different configurations, ranging from the simplest coplanar configuration that involves SPPs propagating along a metal-dielectric interface adjacent to a graphene monolayer, to the most efficient one based on wedge SPPs producing strong electrical fields along a graphene monolayer. To modulate the propagating SPP modes we have used the effect of optical Pauli blocking. This effect is based on the change of graphene optical conductivity induced by the shift of the Fermi energy produced by gating. To completely block optical transitions in graphene at the telecom wavelength of $\lambda=1.5 \mu \mathrm{m}$, one needs to shift the Fermi energy from pristine values of $E_{\mathrm{F}} \approx 0$ to $E_{\mathrm{F}}=\hbar \pi c / \lambda \approx 0.4 \mathrm{eV}$.

\section{Results}

Hybrid graphene plasmonic waveguide geometry. The most straightforward HGPWM geometry modulated by the Pauli blocking effect (Fig. 1a) is based on the classical SPP configuration and is shown in Fig. 1b, top inset, where the gold strip (yellow colour), which supports the SPP propagation and serves as a backgate, is covered by a hexagonal boron-nitride (hBN) flake (purple colour), acting as a dielectric spacer and atomically smooth substrate for graphene, and a graphene flake (black colour). However, the SPP mode in such a waveguide configuration is, away from the surface plasmon resonance in the visible, only weakly bound to the metal surface and features primarily transverse electromagnetic fields, which do not excite currents in graphene, while in-plane fields (which do interact with the graphene in-plane conductivity) are negligibly small in the infrared ${ }^{29}$. Hence, the classical HGPWM configuration, producing only very weak graphene-related absorption (Supplementary Discussion 1A) and promising thereby only very weak modulation by gating, can hardly be used in practice. Attempting to enhance in-plane field components in the graphene layer, we introduced a nanostructured (corrugated) part of the plasmonic waveguide (Methods) so as to produce longitudinal near fields generated by the SPP mode propagating along the corrugated part of waveguide (see middle inset of Fig. 1b). It is however clear that the expected enhancement of in-plane field components is quite limited as the metal surface corrugation has to be shallow to not introduce significant additional propagation losses by scattering. Finally, we decided to make use of the wedge SPP mode supported by the edge of 
a

b
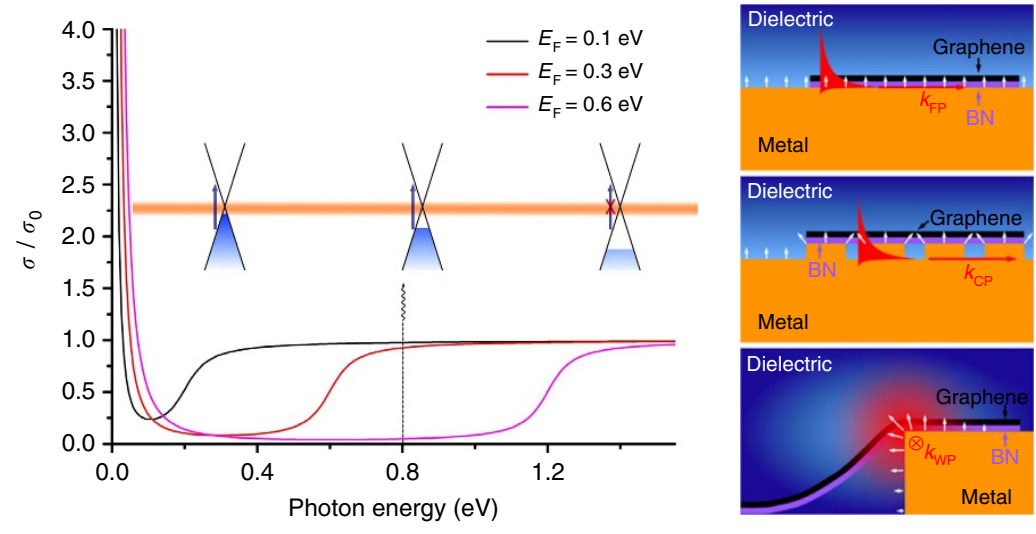

C

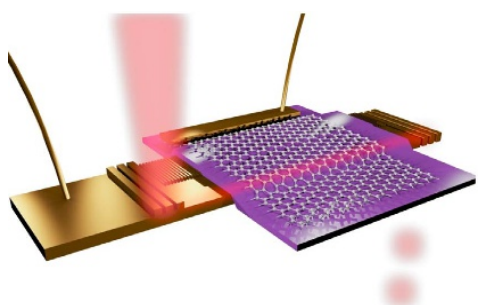

d

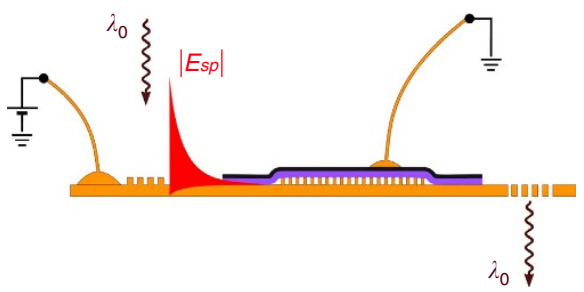

Figure 1 | Principle of hybrid graphene plasmonic waveguide modulators. (a) Optical Pauli blocking expressed in terms of graphene relative conductivity. (b) Sketches of three types of plasmonic modes under investigation-flat, corrugated and wedge plasmons. White arrows indicate approximate direction of electric field. (c) 3D rendering of the experiment with the wedge plasmon mode. (d) The schematic of experiment where non-transparent grating couples light into plasmon modes (flat, corrugated or wedge), which can be affected by gated graphene, black layer, placed on the top of dielectric spacer (a flake of boron nitride, purple layer) and then be decoupled into light through the transparent grating.

planar section of the waveguide 30,31 (see bottom inset of Fig. 1b,c). This mode, in addition to enhanced in-grapheneplane fields near the edge of the strip that should result in higher modulation depth induced by graphene gating (Supplementary Discussions 1 and 5 and Supplementary Fig. 4), has superior field confinement characteristics, which is essential when considering potential applications to the surface plasmon circuitry. Figure $1 \mathrm{~d}$ provides a general outline of modulation experiments: a nontransparent gold grating couples light into a plasmon-propagating mode that can be affected by gated graphene placed on the top of dielectric spacer and then running plasmons are decoupled into light through the transparent grating. Such configuration allows one to decrease the crosstalk between input and output lights, see Methods.

All three studied plasmon-polariton modes-flat plasmons (FPs), corrugated plasmons (CPs) and wedge plasmons (WPs) - can be excited by moving the incident light beam to different parts of the coupler (Fig. 2a). An optical micrograph of one of our devices studied in this work is shown in Fig. $2 b$ along with outlines demonstrating positions of hBN and graphene flakes. We have checked operation of plasmonic waveguides in both transmission and leakage radiation ${ }^{32,33}$ modes. Leakage radiation detection of plasmonic propagating modes for wedge and FPs are shown in Fig. 2c. Figure 2c confirms that the plasmonic modes were successfully excited and propagated along the waveguide. For completeness, Fig. $2 \mathrm{~d}$ provides a scanning electron microscope micrograph of an area marked in Fig. $2 b$ by the blue dashed box where the semitransparent decoupler and a part of the nanostructured area of the waveguide are shown. As preliminary experiments, we performed AFM studies of our samples to find thickness of hBN crystals (which turned out to be $\sim 50-70 \mathrm{~nm}$, see Supplementary Fig. 1 and Supplementary Discussion 2) and hence to deduce the gating voltage necessary to induce optical Pauli blocking at the telecom wavelength $(\sim 10-16 \mathrm{~V})$. We also measured dc graphene resistance as a function of gating voltage in flat and corrugated regions of the waveguide with an idea to evaluate the position of the charge neutrality point $(\mathrm{CNP})$ in graphene $\left(V_{\mathrm{CNP}} \sim 0 \mathrm{~V}\right)$, see Supplementary Fig. 2 and Supplementary Discussion 3. Alternatively, the CNP has been evaluated from Raman measurements described in Supplementary Fig. 3 and Supplementary Discussion 4.

Modulation of plasmonic waveguides by graphene gating. Here we describe the main results of our experiments. The plasmonic waveguides were excited using telecom laser providing $\sim 3 \mathrm{~mW}$ of power at wavelength $\lambda=1.5 \mu \mathrm{m}$. We measured the dynamic response of our modulators by applying an offset square-wave voltage to the back gate with peak-to-peak amplitude $V_{\mathrm{g}}^{\mathrm{pp}}$ and dc component $V_{\mathrm{g}}^{\mathrm{dc}}$. Figure 3 shows the modulation-depth characteristics both as a function of $V_{\mathrm{g}}^{\mathrm{pp}}$ and $V_{\mathrm{g}}^{\mathrm{dc}}$. Comparing the modulation depth of FP and CP modes (Fig. 3a), one can see that the modulation effect is substantially stronger for $\mathrm{CP}$ : the $\mathrm{CP}$ mode gives around an eightfold increase in modulation depth compared with FP (for large $V_{\mathrm{g}}^{\mathrm{dc}}-V_{\mathrm{CNP}}$ ). Here $V_{\mathrm{g}}^{\mathrm{pp}}$ is set to 7.6 and $6 \mathrm{~V}$ for the measurements of the FP and $\mathrm{CP}$ mode modulation depths, respectively. For both FP and CP we see an approximately symmetrical increase in modulation depth with $V_{\mathrm{g}}^{\mathrm{dc}}$, which we attribute to being the positions of the CNP (for the $\mathrm{FP}$ mode this is $V_{\mathrm{CNP}} \approx 2.7 \mathrm{~V}$; for the $\mathrm{CP}$ mode this is $\left.V_{\mathrm{CNP}} \approx 0.9 \mathrm{~V}\right)$. For other samples the modulation for FP modes compared with CP modes was even less pronounced (see Supplementary Fig. 5 and Supplementary Discussion 6). A drastic improvement in the modulation depth is expected from the increased interaction of graphene with electric field of the $\mathrm{CP}$ 
a

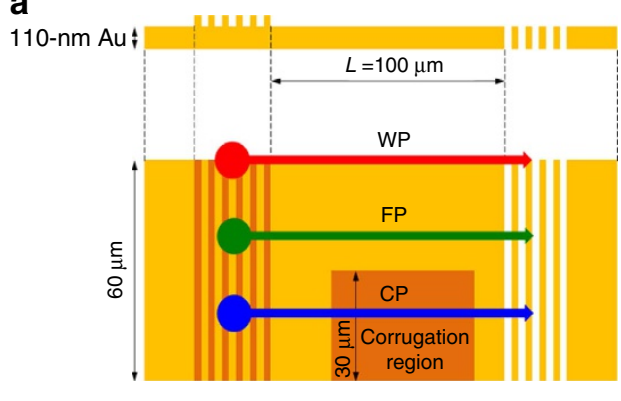

C

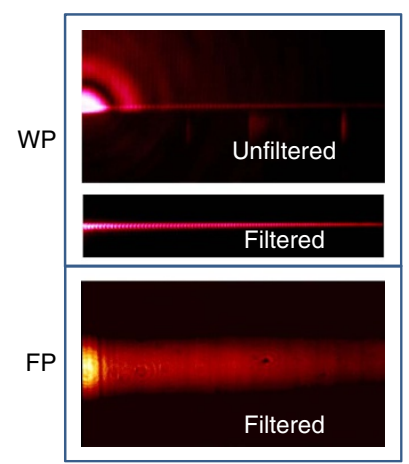

b

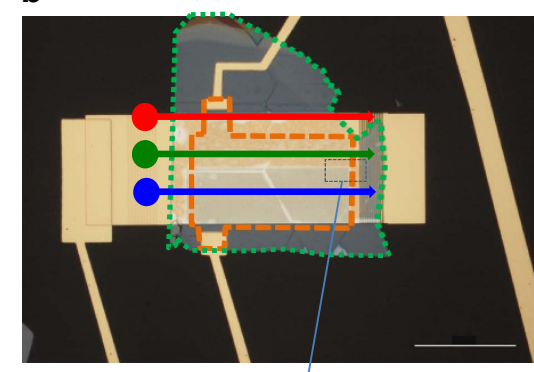

d
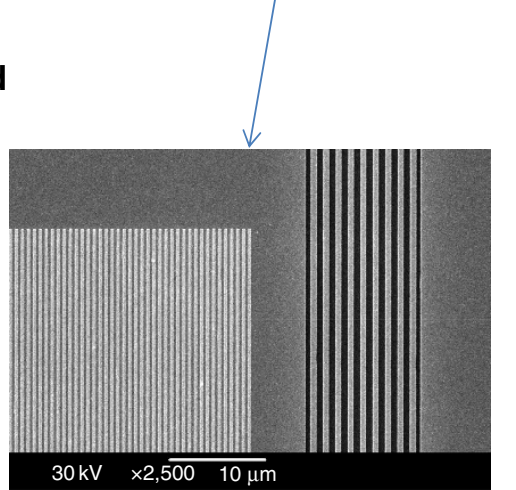

Figure 2 | Plasmon modes of hybrid graphene plasmonic waveguide modulators. (a) The schematics of a studied plasmonic waveguide. The red, green and blue arrows represent WP mode, FP mode and CP mode, respectively. (b) The optical micrograph of a typical hybrid graphene plasmonic modulator studied in this work. The red, green and blue arrows represent WP, FP and CP modes, respectively. An area enclosed by green dotted line represents hBN. An area enclosed by dotted brown line represents graphene. Scale bar, $50 \mu \mathrm{m}$. (c) Leakage radiation detection of wedge, upper panel, and flat, lower panel, plasmon-propagating modes. The wedge mode is given in both raw and Fourier filtered images. (d) A scanning electron micrograph of an area shown in $\mathbf{b}$ by the dotted box that shows corrugated waveguide and the semitransparent decoupling grating.

mode. Indeed, the longitudinal component of the electric field of FP is rather weak, whereas the presence of corrugations creates strong local longitudinal fields near ridges (see the sketch of the mode on Fig. 1b and, for example, ref. 34).

The WP mode provided us with the greatest modulation depths, achieved when probing the top edge of the waveguide, see inset Fig. 3b. Similarly to FP and CP, the modulation depth of WP increases symmetrically from an offset gate voltage $V_{\mathrm{g}}^{\mathrm{dc}}$, which in this case is $\approx 0 \mathrm{~V}$ and is close to $V_{\mathrm{CNP}}$ observed in dc resistivity measurements. For $V_{\mathrm{g}}^{\mathrm{dc}}=6 \mathrm{~V}$ and $V_{\mathrm{g}}^{\mathrm{pp}}=9 \mathrm{~V}$ we were able to achieve a modulation depth of $3.3 \times 10^{-2} \mathrm{~dB}_{\mu \mathrm{m}}^{-1}$, $\sim 30$ and 230 times larger than the best $\mathrm{CP}$ and FP modulation depths with similar set parameters, respectively, Fig. $3 \mathrm{~d}$. It is important to note that the measured modulation depth for WP is underestimated, because, owingto the sample geometry, it was not possible to excite solely the WP mode-the FP mode was always excited on the adjacent flat region. As a result, the detected modulated WP signal had a contribution from FP, which was modulated by a substantially lower degree. A more than an order-of-magnitude increase in the modulation values of WP over CP (Fig. 3b,d) can be again attributed to an increased in-plane (in this case, lateral) electric-field component of the WP mode, which is essential in excitation of currents in graphene (see the sketch of the mode on Fig. 1b and Supplementary Fig. 4 and Supplementary Discussion 5 for a more detailed analysis of the WP mode). The largest modulation depth observed for the WP mode was $\sim 8.7 \%$ for a $12-\mu \mathrm{m}$ modulation length. Note (see Supplementary Discussion 1) that the observed improvement in the modulation depth when changing from FP to WP configuration is quite similar to that estimated for the case of changing

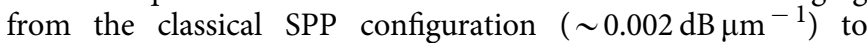
the metal nanowire covered with the flat graphene layer $\left(\sim 0.3 \mathrm{~dB} \mu \mathrm{m}^{-1}\right)$, that is, when exploiting the effect of metal surface curvature resulting in non-zero projections of strong transverse SPP fields on the graphene plane. The largest modulation depth of $0.03 \mathrm{~dB}_{\mu \mathrm{m}}^{-1}$ achieved in our hybrid graphene-plasmonic waveguides is lower than that $\left(0.1 \mathrm{~dB}_{\left.\mu \mathrm{m}^{-1}\right)}\right)$ reported for the hybrid graphene-silicon waveguides $^{15}$. It is worth noting, however, that the silicon waveguide technology is significantly more matured than the plasmonic one. At the same time, our hybrid plasmonic waveguides are already made smaller than analogous silicon ones, while the modulation depth is expected to be considerably improved with a dedicated design.

\section{Discussion}

We found that the dependency on $V_{\mathrm{g}}^{\mathrm{dc}}$ can be described using a simple theoretical model. Since our plasmonic waveguides are uniform along the propagation direction in the region of graphene flake, we can introduce an additional absorption coefficient induced by graphene as $\alpha_{\mathrm{gr}}=\pi \alpha \frac{g^{2} \gamma}{L_{\text {eff }}} \operatorname{Re}\left[\frac{\sigma\left(\omega, V_{\mathrm{g}}\right)}{\sigma_{0}}\right]$, where $\alpha$ is the fine structure constant, $L_{\text {eff }}$ is an effective size of plasmon mode, $\sigma\left(\omega, V_{\mathrm{g}}\right)$ is the gate-dependent graphene optical conductivity and $\sigma_{0}$ is the universal graphene optical conductivity $^{14}$. The additional dissipation of power introduced by the presence of graphene layer can be written as $P=P_{0} \exp \left(-\alpha_{\mathrm{gr}} l\right)$, where $l$ is the length of the waveguide. Using the Kubo expression for graphene conductivity and writing Fermi energy as $E_{\mathrm{F}}=\operatorname{Sign}\left(\Delta V_{\mathrm{g}}\right) \hbar v_{\mathrm{F}} \sqrt{\pi C\left|\Delta V_{\mathrm{g}}\right| / e}$, where $\Delta V_{\mathrm{g}}=V_{\mathrm{g}}-V_{\mathrm{CNP}}, v_{\mathrm{F}}$ is the Fermi speed in graphene and $C$ is the gate capacitance, we were able to model the modulation data obtained in our experiments. The modelling results (the solid lines of Fig. 3c,d) 
a

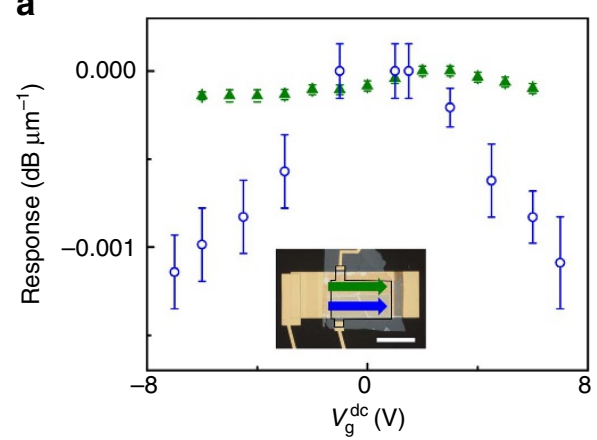

C

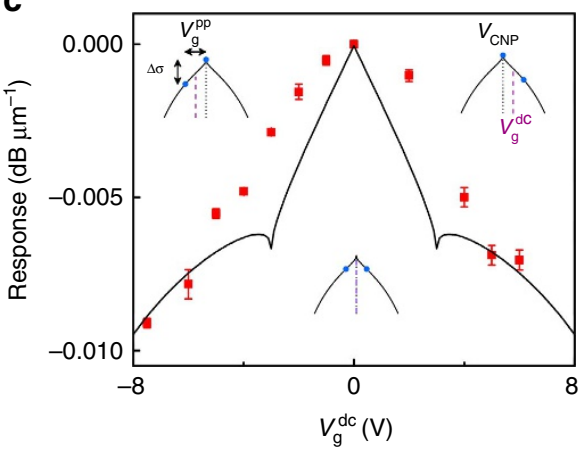

b

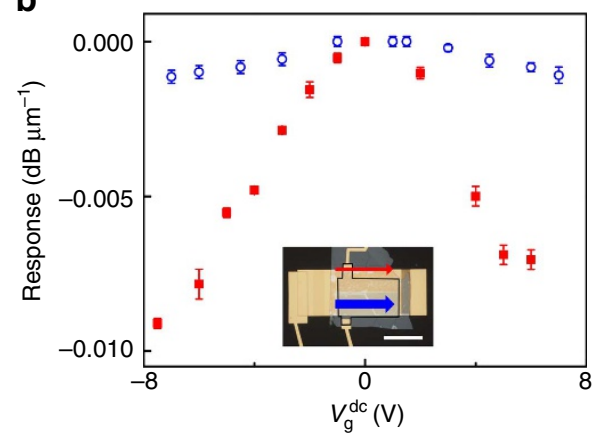

d

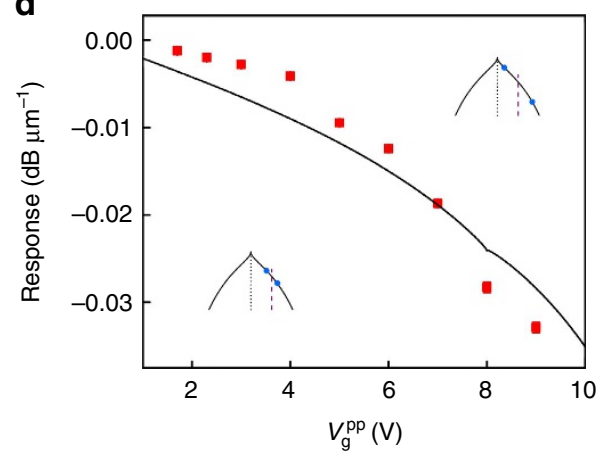

Figure 3 | Operation of hybrid graphene plasmonic waveguide modulators. (a) Modulated $A C$ transmission of the waveguide expressed in $\mathrm{dB}_{\mu \mathrm{m}}{ }^{-1} \mathrm{as}$ a function of gating voltage for the flat, green data points, and the corrugated, blue data points, plasmon modes. The peak-to-peak amplitude of AC modulation was 7.6 and $6 \mathrm{~V}$ for FP and CP modes, respectively, the frequency $6 \mathrm{~Hz}$. The filled and empty data points represent two different devices. The inset shows the position where the plasmonic modes were measured. (b) Modulated $A C$ transmission of the waveguide expressed in $\mathrm{dB}_{\mu} \mathrm{m}^{-1} \mathrm{as}$ a function of gating voltage for the wedge, red data points, and the corrugated, blue data points, plasmon modes. The amplitude of AC modulation was $6 \mathrm{~V}$, the frequency $6 \mathrm{~Hz}$. Notice the 10 -fold increase of the modulation signal for wedge mode. The filled and empty data points represent two different devices. The inset shows the position where the plasmonic modes were measured. (c) Theoretical fit to the experimental data for the wedge mode modulation as a function of gating obtained for the following parameters: peak-to-peak modulation amplitude $6 \mathrm{~V}$, frequency $6 \mathrm{~Hz}$. (d) Modulated AC transmission of the waveguide expressed in $\mathrm{dB}_{\mu \mathrm{m}}^{-1}$ as a function of $\mathrm{AC}$ peak-to-peak amplitude for the wedge plasmon mode, red data points, and theoretical fit to the experimental data. The dc offset was $6 \mathrm{~V}$, the frequency $6 \mathrm{~Hz}$. Error bars were estimated as a noise level in the absence of $A C$ modulation and were combined with random errors whenever repeated measurements were performed.

match our experimental data well. Insets in Fig. 3c,d provide a simplified picture of the change of modulation with gating. The square-wave gate voltage results in a switching between two optical conductivities, with the difference $\Delta \sigma$ dependent on $V_{\mathrm{g}}^{\mathrm{dc}}$ and $V_{\mathrm{g}}^{\mathrm{pp}}$ (see insets for Fig. $3 \mathrm{c}, \mathrm{d}$ ). When $V_{\mathrm{g}}^{\mathrm{dc}}=V_{\mathrm{CNP}}$, there is a switching between electron and hole conductivity, yet the magnitude of the absolute conductivity of graphene (and therefore transmission through the waveguide) is constant, and so the modulation depth is zero. As we move far enough from $V_{\mathrm{CNP}}, \Delta \sigma$ generally increases with $\left|V_{\mathrm{g}}^{\mathrm{dc}}-V_{\mathrm{CNP}}\right|$ that leads to the observed dependency on $V_{\mathrm{g}}^{\mathrm{dc}}$. The scale of the modulation depth about $V_{\mathrm{CNP}}$ is then set by the magnitude of the coupling of graphene with the in-plane electric field of the plasmonic mode. Similar arguments explain the increasing modulation depth with $V_{\mathrm{g}}^{\mathrm{pp}}$ (Fig. $3 \mathrm{~d}$ ), when $V_{\mathrm{g}}^{\mathrm{dc}}$ is set away from the CNP.

In conclusion, exploiting the enhancement of in-grapheneplane plasmonic fields provided by the wedge SPP waveguide configuration, we have demonstrated a prospective hybrid graphene-plasmonic waveguide modulator working at telecom wavelengths with reasonably high modulation depth of $>0.03 \mathrm{~dB} \mu \mathrm{m}^{-1}$ induced by a moderate gating voltage of $<10 \mathrm{~V}$. The modulator gating voltage can be reduced to several volts for hBN thickness of $10-20 \mathrm{~nm}$. To achieve low gating voltages it is also important to have homogenous graphene flakes (that have constant doping along the flake surface). Note that the achieved modulation depth exceeds by $\sim 50 \%$ the intensity modulation depth corresponding to the highest phase modulation demonstrated with the state-of-the-art plasmonic modulator ${ }^{35}$. The frequency of the modulation is limited by the time constant of the structure ${ }^{1,15}$ and can be evaluated at the level $>1 \mathrm{GHz}$. Plasmonic hybrid waveguides with a smaller physical size can provide even larger modulation speeds of $>65 \mathrm{GHz}$ (ref. 35). Therefore, one might expect that a waveguide based on cylindrical SPP (Supplementary Discussion 1C) could be modulated by graphene gating at frequencies of $\sim 100 \mathrm{GHz}$.

The use of wedge SPP waveguide configuration for light modulation by graphene gating thereby paves the way towards practical realization of very compact and efficient, potentially ultrafast $^{1}$ and broadband ${ }^{11}$ hybrid graphene-plasmonic optical devices for a wide range of applications in optoelectronics and telecommunications.

\section{Methods}

Plasmonic waveguides. The gold plasmonic waveguides were fabricated in two lithography steps. In the first step of electron-beam lithography (and subsequent lift-off), we fabricated a plasmonic waveguide-a golden strip of $110 \mathrm{~nm}$ thickness, $100 \mu \mathrm{m}$ length and $60 \mu \mathrm{m}$ width-and the de-coupler, which was formed of a transparent gold grating for the period of $1.24 \mu \mathrm{m}$ on the top of a quartz substrate. In the second step, we fabricated periodic lateral ridges along the bottom half of the waveguide (covering half of the width and the whole of the length of the gold strip) and the coupling non-transparent gold grating. The coupling grating was optimized for $1.5 \mu \mathrm{m}$ wavelength normal incident light. This configuration of non-transparent coupling grating and transparent de-coupling grating allows 
one to excite the plasmon from one side and to measure the transmitted light from the other side, decreasing the crosstalk between input and output lights. Plasmons propagating along the nanostructured half of the waveguide, referred to as $\mathrm{CP}$, hop over the ridges (arranged in a 600-nm-period grating) and generate in-plane electric fields, whereas along the planar section the evanescent electric field associated with the propagating FPs remains mostly transverse (Fig. 1b). We also studied the WP mode, which exists at the edge of planar section of the waveguide. Coupling of light and launching of plasmons was possible across the whole lateral dimension of the coupler, which allowed us to select the path of the propagating plasmons, see Fig. 2, and excite three different waveguide plasmon modes described above. Error bars in the experimental data were estimated as a noise level in the absence of AC modulation and were combined with random errors whenever repeated measurements were performed.

Graphene preparation. To fabricate the modulation unit on the top of plasmonic waveguides, we applied the micromechanical cleavage technique to produce graphene and hBN flakes on oxidized silicon wafers. Then, hBN crystals and graphene were wet-transferred and aligned on to the waveguide. Oxygen plasma was used to shape graphene to achieve regular geometry of flakes across the waveguide. Here hBN acts as an atomically flat substrate for graphene as well as the gate dielectric. The gold waveguide was used both as the carrier of propagating plasmons and backgate for graphene gating. Finally, we fabricated electrical contacts to graphene (two terminals to allow for electrical characterization of our devices) and to the waveguide/backgate using photolithography. In this study we provide results obtained on two particular devices, with hBN thicknesses $t \approx 50$ and $70 \mathrm{~nm}$ and nanostructure ridge widths of $w=300$ and $200 \mathrm{~nm}$, respectively. Both had a nanostructure ridge pitch of $600 \mathrm{~nm}$.

\section{References}

1. Xia, F. N., Mueller, T., Lin, Y. M., Valdes-Garcia, A. \& Avouris, P. Ultrafast graphene photodetector. Nat. Nanotechnol. 4, 839-843 (2009).

2. Mueller, T., Xia, F. N. A. \& Avouris, P. Graphene photodetectors for high-speed optical communications. Nat. Photon. 4, 297-301 (2010).

3. Echtermeyer, T. J. et al. Strong plasmonic enhancement of photovoltage in graphene. Nat. Commun. 2, 458 (2011).

4. Britnell, L. et al. Strong light-matter interactions in heterostructures of atomically thin films. Science 340, 1311-1314 (2013).

5. Schedin, F. et al. Detection of individual gas molecules adsorbed on graphene. Nat. Mater. 6, 652-655 (2007).

6. Kravets, V. G. et al. Singular-phase nanooptics: towards label-free single molecule detection. Nat. Mater. 12, 304-309 (2013).

7. Shao, Y. et al. Graphene based electrochemical sensors and biosensors: a review. Electroanalysis 22, 1027-1036 (2010).

8. Bonaccorso, F., Sun, Z., Hasan, T. \& Ferrari, A. C. Graphene photonics and optoelectronics. Nat. Photon. 4, 611-622 (2010).

9. Grigorenko, A. N., Polini, M. \& Novoselov, K. S. Graphene plasmonics. Nat. Photon. 6, 749-758 (2012).

10. Ju, L. et al. Graphene plasmonics for tunable terahertz metamaterials. Nat. Nanotechnol. 6, 630-634 (2011).

11. Li, Z. Q. et al. Dirac charge dynamics in graphene by infrared spectroscopy. Nat. Phys. 4, 532-535 (2008).

12. Wang, F. et al. Gate-variable optical transitions in graphene. Science 320, 206-209 (2008)

13. Zhang, Y. B. et al. Direct observation of a widely tunable bandgap in bilayer graphene. Nature 459, 820-823 (2009).

14. Nair, R. R. et al. Fine structure constant defines visual transparency of graphene. Science 320, 1308-1308 (2008).

15. Liu, M. et al. A graphene-based broadband optical modulator. Nature 474, 64-67 (2011)

16. Kim, K., Choi, J. Y., Kim, T., Cho, S. H. \& Chung, H. J. A role for graphene in silicon-based semiconductor devices. Nature 479, 338-344 (2011).

17. Liu, M., Yin, X. \& Zhang, X. Double-layer graphene optical modulator. Nano Lett. 12, 1482-1485 (2012).

18. Li, W. et al. Ultrafast all-optical graphene modulator. Nano Lett. 14, 955-959 (2014).

19. Youngblood, N., Anugrah, Y., Ma, R., Koester, S. \& Li, M. Multifunctional graphene optical modulator and photodetector integrated on silicon waveguides. Nano Lett. 14, 2741-2746 (2014).

20. Geim, A. K. \& Novoselov, K. S. The rise of graphene. Nat. Mater. 6, 183-191 (2007)
21. Yan, H. et al. Tunable infrared plasmonic devices using graphene/insulator stacks. Nat Nano 7, 330-334 (2012).

22. Papasimakis, N. et al. Graphene in a photonic metamaterial. Opt. Express 18, 8353-8359 (2010).

23. Kravets, V. G. et al. Surface hydrogenation and optics of a graphene sheet transferred onto a plasmonic nanoarray. J. Phys. Chem. C 116, 3882-3887 (2012)

24. Kravets, V. et al. Graphene-protected copper and silver plasmonics. Sci. Rep. 4, 5517 (2014).

25. Kim, J. et al. Electrical control of optical plasmon resonance with graphene. Nano Lett. 12, 5598-5602 (2012).

26. Thackray, B. D. et al. Super-narrow, extremely high quality collective plasmon resonances at telecom wavelengths and their application in a hybrid graphene-plasmonic modulator. Nano Lett. 15, 3519-3523 (2015).

27. Wang, H., Wu, Y., Cong, C., Shang, J. \& Yu, T. Hysteresis of electronic transport in graphene transistors. ACS Nano 4, 7221-7228 (2010).

28. Ju, L. et al. Photoinduced doping in heterostructures of graphene and boron nitride. Nat. Nano 9, 348-352 (2014).

29. Raether, H. in Surface Plasmons on Smooth and Rough Surfaces and on Gratings, Springer Tracts in Modern Physics Vol. 111 (Springer, 1988).

30. Pile, D. F. et al. Theoretical and experimental investigation of strongly localized plasmons on triangular metal wedges for subwavelength waveguiding. Appl. Phys. Lett. 87, 061106 (2005).

31. Moreno, E., Rodrigo, S. G., Bozhevolnyi, S. I., Martín-Moreno, L. \& García-Vidal, F. Guiding and focusing of electromagnetic fields with wedge plasmon polaritons. Phys. Rev. Lett. 100, 023901 (2008).

32. Radko, I. P., Fiutowski, J., Tavares, L., Rubahn, H.-G. \& Bozhevolnyi, S. I. Organic nanofiber-loaded surface plasmon-polariton waveguides. Opt. Express 19, 15155-15161 (2011).

33. Drezet, A. et al. Leakage radiation microscopy of surface plasmon polaritons. Mater. Sci. Eng. 149, 220-229 (2008).

34. Radko, I. et al. Efficient unidirectional ridge excitation of surface plasmons. Opt. Express 17, 7228-7232 (2009).

35. Melikyan, A. et al. High-speed plasmonic phase modulators. Nat. Photon. 8, 229-233 (2014).

\section{Acknowledgements}

A.N.G., D.A. and F.J.R. are grateful to the support by SAIT GRO Program, EPSRC grant EP/K011022/1, Bluestone Global Inc. Grant, E.C. Graphene Flagship grant (contract no. CNECT-ICT-604391). I.P.R., Z.H. and S.I.B. acknowledge financial support for this work from the Danish Council for Independent Research (the FTP project ANAP, Contract No. 09-072949) and from the European Research Council, Grant No. 341054 (PLAQNAP).

\section{Author contributions}

S.I.B. and A.N.G. conceived the experiment, devised waveguides and geometry of modulators. Z.H. and I.P.R. fabricated plasmonic waveguides, conducted their optical characterization and optimized them. D.A. and F.J.R. devised and fabricated electrical part of the devices and conducted their electrical, AFM and Raman characterizations. D.A., F.J.R. and I.P.R. characterized the performance of fabricated modulators and processed the experimental data. All authors discussed the results and participated in preparing the manuscript.

\section{Additional information}

Supplementary Information accompanies this paper at http://www.nature.com/ naturecommunications

Competing financial interests: The authors declare no competing financial interests.

Reprints and permission information is available online at http://npg.nature.com/ reprintsandpermissions/

How to cite this article: Ansell, D. et al. Hybrid graphene plasmonic waveguide modulators. Nat. Commun. 6:8846 doi: 10.1038/ncomms9846 (2015).

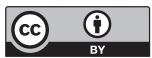

This work is licensed under a Creative Commons Attribution 4.0 International License. The images or other third party material in this article are included in the article's Creative Commons license, unless indicated otherwise in the credit line; if the material is not included under the Creative Commons license, users will need to obtain permission from the license holder to reproduce the material. To view a copy of this license, visit http://creativecommons.org/licenses/by/4.0/ 